\title{
Technical constraints in the development of reduced-fat bakery products
}

\author{
Terry Sharp \\ Campden and Chorleywood Food Research Association, Chipping Campden, Glos. GL55 6LD, UK
}

\begin{abstract}
The present study was funded by the Ministry of Agriculture, Fisheries and Food, its aim being to identify the technical barriers to the development of reduced-fat alternatives for bakery products. Using National Food Survey (Ministry of Agriculture, Fisheries and Food, 1998) statistics on dietary consumption within the home, biscuits, cakes and pastries were identified as contributing significant amounts of fat to the population's dietary intake. Face-to-face interviews were conducted with contacts in the technical community of the baking industry, who were usually working in technical and new-product development functions. A discussion guide was developed to cover the main lines of enquiry. The companies selected were ingredient suppliers (eight), manufacturers (twelve) and retailers (four) and so represented each step of the food supply chain. In brief, results showed that current labelling rules were too stringent, and constrained development of reduced-fat bakery products. Products with lower fat levels are harder to make due to altered handling and processing properties. Their quality is usually poorer than standard products, particularly for flavour, texture and mouthfeel. The perception of freshness is reduced and product shelf-life may consequently be shorter. For the product developer, there are relatively few ingredients that can be used in place of fat, and knowledge of how they work is limited, which inhibits product development. There is no identifiable source of technical knowledge in this field. Consumers perceive reduced-fat bakery products to be of lower quality and are generally unwilling to pay higher prices than for standard products.
\end{abstract}

Reduced-fat bakery products: Food labelling: Fat substitutes: New product development: Consumer response

Biscuits, cakes and pastries contribute significant amounts of fat to the UK dietary intake, as shown by the National Food Survey. The present study, funded by the Ministry of Agriculture, Fisheries and Food, aimed to identify the technical barriers to the development of reduced-fat alternatives for this type of bakery product. The project was part of a larger study, which included the consumer perceptions, and attitudes related to reduced-fat bakery products (RFBP; defined as biscuits, cakes or pastries having a fat content that is below that of a similar standard product).

\section{Methods}

Using National Food Survey (Ministry of Agriculture, Fisheries and Food, 1998) statistics on dietary consumption within the home, biscuits, cakes and pastries were identified as contributing significant amounts of fat to the population's dietary intake.

Face-to-face interviews, usually lasting $1-2 \mathrm{~h}$, were conducted with contacts in the technical community of the baking industry. The companies selected were ingredient suppliers, manufacturers and retailers, and so represented each step of the food supply chain. Two internationallyrecognised experts in the bakery product and fat-reduction field were also contacted since they are key contacts for those in industry who are trying to develop RFBP. Table 1 gives a breakdown of the type of contacts approached.

\section{Study structure}

A discussion guide was developed to cover the main lines of enquiry. Information was gathered by face-to-face discussions with identified contacts. A series of 'anchor' questions were developed to form the direction for various lines of questioning. This approach ensured that the interviewers were able to cover all areas of interest for the survey. These questions were:

1. what sort of products does your company make;

2. how is your current range changing; 
Table 1. List of company contacts by type

\begin{tabular}{lrrrr}
\hline & \multicolumn{2}{c}{ No. of companies participating } & \\
\cline { 2 - 5 } & Large & Medium & Small & No. of contacts \\
\hline Ingredient suppliers & 6 & 2 & - & 9 \\
Producers & 4 & 5 & 3 & 15 \\
Retailers & 4 & - & - & 8 \\
Experts & - & - & - & 2 \\
Totals & 14 & 7 & 3 & $34^{*}$ \\
\hline
\end{tabular}

* Contacts were working in technical, product development, sales applications and nutrition functions within their companies, who were all members of Campden and Chorleywood Food Research Association.

3. is nutrition taken into account when developing RFBP;

4. how difficult is RFBP development;

5. what level of knowledge exists to aid RFBP development;

6. how do you see consumer reaction to RFBP;

7. do RFBP have a long-term future.

Interviews, usually lasting $1-2 \mathrm{~h}$ were conducted either at the Campden and Chorleywood Food Research Association site, or at the company itself. Of the thirty-four total contacts, seventeen interviews were conducted with one individual, seven with two individuals and one with three individuals. They were conducted between June and September 1998 by Dr TM Sharp and Mr PF Catterall of the Campden and Chorleywood Food Research Association.

\section{Confidentiality}

Since some areas of product design and development may be commercially sensitive, it was agreed with all respondents that any information given or discussed would be used in ways which will not be attributable to individuals or their companies. No company, brand or personal names have therefore been used in this report, except where information is already freely available in the public domain, for instance products on sale to the public.

\section{Results}

All comments recorded were the personal and professional views of the contacts interviewed. Although there were large areas of common ground, particularly in relation to the barriers that prevent further development of RFBP, occasionally contradictory information was received. The present report attempts to reflect all views, since the authors consider that all are equally valid. Comments are recorded under the main headings from the questionnaire.

\section{What sort of products does your company make?}

\section{Standard products}

Ingredient suppliers. Bakery mixes, bakery creams and fillings, emulsifiers, egg products, bulking agents, fruit syrups, starches, stabilisers, fibre and soyabean protein.
Manufacturers and retailers. Cream cakes, layered desserts, chilled and frozen gateaux, Christmas puddings, celebration and novelty cakes, fruit and plain cakes, sponge sandwich and bar cakes.

Sweet and savoury pies, short and puff pastry, eclairs, cream slices, Danish pastries, spiced and fruited buns, filled flans and tarts, and steamed puddings.

Fig rolls, digestive type, mallow, shortcake, iced and chocolate-covered biscuits, cream-filled biscuits, Rich Teatype, custard creams, malted milk, Gingernut-type, bourbon and chocolate-covered countlines.

Cream crackers, water biscuits, crispbreads, Ritz-type crackers, cheeselets, grain crackers and Twiglet-type snacks.

\section{Reduced-fat products}

Ingredient suppliers. Reduced fat: cake premixes, creams, triacylglycerols, milk and cream (liquid and dry).

Ingredients to help reduce fat: emulsifiers, gums, starches, fruit preparations, fibre and soyabean, egg and dairy proteins.

Manufacturers and retailers. Cakes: plain and topped cakes, chocolate cakes, hot cross buns, Danish pastries, assembled cake-based desserts, hot and cold pies, eclairs.

Biscuits: digestive (plain and coated), shortcake, custard cream, fig rolls, cream crackers.

\section{Notes}

1. All retailers and most other contacts already sell some reduced-fat products.

2. The range is relatively small compared with that of products containing fat at standard levels. RFBP:standard products of 1:20 was quoted by one respondent. Another respondent quoted the production volume of a reduced-fat alternative to be $6 \%$ of the standard-fat product.

3. Most products are in the reduced-fat rather than lowfat range.

4. Low-fat products are more common with cakes than biscuits.

5. Some retailer and private label RFBP are based on standard-fat alternatives.

6. Private label branded ranges are being sold which are based on claims for fat contents, i.e. ' $\mathrm{x} \%$ fat free'. Examples, found in most retailers are the brands 'Go Ahead' (United Biscuits (UK) Ltd, London, UK), 'Vitalinea' (The Jacob's Bakery Ltd, Liverpool, UK) and 'Weight-Watchers' (HJ Heinz Co Ltd, Uxbridge, UK).

\section{How is your current range changing?}

\section{New product development}

Across the range of cakes, biscuits and pastries it is clear that development in the reduced-fat area is taking place, although there was a variable response concerning the number of new products. Generally, the retailers expect to launch new RFBP (in one case doubling the current range), 
although the more common view was that there would be a small increase in product numbers. These products will not necessarily come from all the producers interviewed, since some producers had no immediate plans for new RFBP whereas other producers were planning individual products or even new ranges or range extensions.

\section{The stimulus for new products}

Throughout the supply chain it is common for the stimulus for development to come from the customer-supplier relationship:

\section{consumers influence retailers}

retailers influence manufacturers

manufacturers influence ingredient suppliers

The influences are not this simple. Retailers also approach ingredient suppliers, and retailers are also influenced directly by media issues on nutrition and health, and indirectly by the media influence on the consumer. Retailers in particular also showed a high awareness of Government policy, and appear to use this policy in developing parts of their own product strategy. It also emerged that influence can be two-way, so that partnerships for product development are built between retailers and manufacturers. Further, the stimulus is often internal and is driven by real or perceived competition in the market place. Thus, manufacturers do also take the initiative in developing new product ranges to increase their market share and turnover, and retailers may offer these product ranges, and their own new products to secure an advantage over their competitors.

From discussions with retailers it also emerged that company nutritionists play an important role by interfacing between Government policy on health and nutrition and the development of products to meet policy requirements.

\section{Is nutrition taken into account when developing reduced-fat baking products?}

\section{Are you aware of Government health policy and nutrition targets and are you aware of current regulations for reduced-fat claims?}

Overall there was a very high level of awareness of both the Government's Health and Nutrition Policy (as defined in The Health of the Nation White Paper; Department of Health, 1991) and current regulations for claims for RFBP, the only exceptions being suppliers of specialist ingredients who were somewhat removed from the process of new product development. A number of respondents commented that awareness of both topics was very low amongst consumers. The general comment was that most developers are interested in the quantity of fat in their products; although some consider fat quality, this factor is not the major issue.

\section{Do labelling regulations and guidelines help or hinder development of reduced-fat baking products?}

There was no concensus here. Six respondents believed that the regulations and guidelines for reduced-fat or low-fat claims (Food Advisory Committee, 1989) were helpful, since they provide a consistent framework for claims and enable clear and simple messages to be given on product packs. For other respondents, this factor was a problem rather than a benefit, since information which can be given to the consumer is too tightly controlled. An example given was that it is not possible to declare on the front of a pack that the product within contains, for example, $10 \%$ fat, yet it may be helpful for a purchaser to know, in simple terms, the fat content of products. They could then make quick and informed choices without having to refer to the nutritional panel. In itself, this simple change could enable consumers to reduce their fat intakes by easier product selection when shopping for food.

A number of respondents commented that the level of fat reduction needed to allow a reduced-fat claim (25\%) was too difficult a target considering the technical challenges involved. The overall effect is to limit the number of RFBP available to consumers, which in turn reduces the opportunity to decrease fat intakes by this route. One expert view was that changing the value to a $10 \%$ reduction of fat to trigger a claim would encourage the introduction of more RFBP, and therefore reduce consumption of fat.

The reference point from which fat is reduced was thought by some respondents to cause difficulty for two reasons. First, it is not possible to define a standard fat level for a product, and second, as more RFBP appear, the reference point itself could change, i.e. the reduced-fat version could become the new standard. In this context, reduced-fat milk (i.e. semi-skimmed or skimmed) was given as an example.

Some producers mentioned that legislation could lag behind developments. For example, many products have appeared which are a mixture of different components and so do not fit the criteria for certain types of product. An example is a layered dessert that contains cake, but cannot be classified as a cake.

A comment was also made about the criteria for low-fat claims which require a fat content of less than $5 \%$. (This project was completed before the Food Advisory Committee (1999) revised its guidelines for low-fat claims.) Since many ingredients used already contain some fat (e.g. flour) the target for a low-fat claim can be very difficult to achieve. It may be more helpful to make a claim based on 'added fat' to help overcome this problem.

The use of ' $x \%$ fat-free' claims was not fully supported. On one hand, the view was that these claims are more easily understood by consumers, but some respondents believe that they are misleading, giving the impression that products may be nearly free of fat, whilst in reality still containing reasonable levels.

Finally, there were a number of comments about the confusion which exists between various claims, even with the clear regulations and guidelines which are in place. These comments are related to the motivation of consumers to purchase products with reduced- or low-fat claims. It may be that at times consumers will buy RFBP in the belief that they contain less energy and, therefore, will help with weight control, rather than thinking about the effect of fat on diet and health. The criteria for reduced- and low-fat claims do not at all guarantee less energy in the product; in fact, other ingredients which are used in place of fat 
often contribute energy to the product to the same extent as fat.

Two related questions in the discussion guide were: 'do you always make claims for reduced-fat products' and 'would you reduce the fat content if no claim could be made'. The answers are relevant here, but cover a number of issues. It was noted that there must be a justification to reduce the amount of fat in a product. This justification may be economic if a cheaper ingredient can be used instead, or technical if a quality improvement can be achieved. However, the response generally was that if there was no technical or economic justification, and no claim could be made for nutrition, the fat content would not be reduced. The conclusion is that an opportunity is being lost for consumers to purchase products that have small reductions in fat content. Very few of those questioned responded positively to the idea of incrementally reducing fat contents without making claims.

\section{What more can Government do to encourage development of reduced-fat bakery products?}

The main conclusion was that Government could help further with various aspects of consumer education. In particular, consumers should be made more aware of Government policy and targets for health and dietary intakes. The point was made that although Government publicises the output from the various committees such as the Committee on Medical Aspects of Food Policy, the output is rarely transferred simply and accurately through the media to the consumer. Clearer targeted information would be useful to enable consumers to make more informed purchases. Education on the fat contents of various foods, and clearer and simpler nutrition information on food packaging would help make the choice of RFBP easier for consumers.

Again, on the same theme, it was mentioned that the industrial sector provides a great deal of nutrition and health information to consumers, but that the level of detail and clarity of the messages can be variable and sometimes may be contradictory. A stronger input from Government could help reinforce clearer messages. A number of comments were also made about the opportunity for Government to encourage the educational process at school age; for instance, by including healthy cooking and eating in the curriculum.

Finally, two other points were made. First, regulatory approvals for new ingredients to replace fat are generally slow, possibly inhibiting progress in the development of new RFBP, and second, Government could also stimulate this area by funding more research on the role of fat in foods and the understanding of the functionality of fat substitutes.

\section{How difficult is the development of reduced-fat bakery products?}

This group of questions formed the core of the survey and was designed to explore the technical and economic barriers that may restrict the development of RFBP. Since there is a great deal of information, it is presented as technical (production, texture and flavour, shelf-life and overall quality) and economic.

\section{Technical}

Production. The issues mainly relate to biscuits, since the reduction of fat content appears to affect the properties of doughs more than cake batters. Many actual differences were listed for the manufacture of products containing lower fat levels. The comments are qualitative and may not all lead to equivalent problems during production.

Generally, reducing the fat content of doughs changes their handling properties. Reduced-fat doughs are firmer, tougher and can be stickier, particularly if high sugar levels are present. These properties lead to handling and machining problems during production; in particular, release from equipment surfaces is more difficult, and it may be necessary to use more release agents, or even Teflon coatings on contact surfaces. The main cause is the loss of lubrication when dough fat is reduced.

Reduced-fat doughs are also more abrasive, causing higher levels of wear; for instance, the moulds used for shaping certain short doughs need to be replaced more frequently. For hard doughs, using less fat leads to more gluten development during mixing and consequently greater shrinkage of dough pieces after forming. It may then be necessary to add more processing aids to ensure that the dough is more workable during production. This situation is also true for shortcrust pastry. Also, during hot weather reduced-fat dough toughens more quickly and is more difficult to form into pieces for baking.

For reduced-fat biscuits it is necessary to change a number of steps in the manufacturing process, including recipe balance, mixing and baking. Since reduced-fat doughs are firmer, more water is added to the recipe. All added water is bound more tightly in the dough and the product must be baked at a higher temperature and/or for longer to achieve the final product colour and moisture content.

Reduced-fat doughs are less tolerant to varying process conditions, and it was reported that there is a higher level of quality control failures and up to $1 \%$ more wastage than standard products. Since reduced-fat doughs have different handling properties it is less easy to switch between reduced-fat and standard production. Changeovers take longer, since adjustments have to be made to the production plant. Operators may require additional training to deal with the extra problems that are connected to the manufacture of RFBP. Production runs of RFBP are usually shorter, since sales volumes are lower, and this factor can cause other difficulties. It was reported that a reduced-fat biscuit product could not be made by a particular manufacturer since the standard production line was too big for such a short run.

A comment was made about oil spraying which is a finishing process used to impart colour and flavour to baked crackers and biscuits. Some current equipment in use does not always deliver a consistent amount of oil to the product. If a low-fat claim is being made, the varying amount of added oil could take the total fat content beyond the $5 \%$ maximum, which allows the claim to be made. 
Finally, the factory may need to carry stocks of a wider range of ingredients since non-standard ingredients are often needed to replace fat.

Texture and flavour. There were unanimous views that reducing the fat content of cakes, biscuits and pastries can affect the eating quality of the products. For cakes, reducing the fat content destabilises the batter foaming properties and leads to collapse and loss of volume during baking, which will not only affect appearance but also produce a denser crumb, giving a firmer eating quality. Even if the reduction of fat is not so great as to cause this problem, the removal of fat makes the cake drier and firmer, and less tender, with a shortness of texture. Breakdown of the crumb during eating is also different, and the cake is more difficult to clear from the mouth by swallowing. Colour and flavour also are less good than in the standard product. Reduced-fat biscuits are drier, too crisp and gritty and less easy to swallow, and also suffer a loss of colour and flavour. In pastry, reducing fat content causes a loss of crispness and lift, and increased dryness, with a more brittle and dense texture.

There are particularly difficult problems in reducing the fat level in products that contain high amounts of butter, since its unique flavour and melting profile are very difficult to match with other ingredients. It was also noted that the use of butter and its declaration is a good selling point regarding the quality of the product.

Overall these texture and flavour changes are seen as a matter of degree, so that as more fat is removed the differences from standard products become greater. The general view is that a $10 \%$ reduction in fat levels will only cause minor changes to product quality, but in the more sensitive products that do not contain much moisture, even $25 \%$ reduction can cause easily-detectable changes. For products which contain naturally high levels of moisture, e.g. some types of cakes, it may be possible to reduce fat content by up to $50 \%$ with fewer effects on product quality.

Shelf-life. Biscuits have very good keeping qualities and do not change much during storage, so that reducing their fat content has little effect on shelf-life. If anything, there may be a beneficial effect, since when products contain less fat they are less prone to the development of off flavours due to rancidity.

One storage problem that was mentioned is the defect known as 'checking'. This defect is the spontaneous cracking of biscuits during storage which is due, not through any physical shock, but rather because of internal stresses set up in the biscuit during baking and cooling. It was noted that the difference in water-binding properties of reducedfat doughs could make these products more susceptible to this problem.

Cakes, however, rely on softness and tenderness to create the impression of freshness, and these eating qualities are negatively affected by reducing fat content. Not only does the rate of staling increase when less fat is present in the product, respondents also commented that reduced-fat cakes start out drier and firmer, with less flavour than standard products. The perception is that they are already somewhat stale. Their usable shelf life is thus decreased, which gives problems in the retail distribution and storage chain.

Fillings, toppings and coatings are often an integral part of cakes, biscuits and pastries, and are themselves often fat based. There were mixed views about the ability to reduce their contribution to product fat content. Some respondents have reformulated, for example, chocolate coatings to contain less fat, although compositional standards must still be met. Other respondents have taken the approach of maintaining the composition of creams and toppings and simply reducing the amount used when they have a higher fat content than the biscuit, pastry or cake with which they are combined. One point was raised about reducing the fat content of fillings and toppings. If this process leads to a higher water activity, moisture migration between wet and dry components may affect both eating quality and shelflife.

Overall quality. A number of respondents commented that the single overall effect of removing fat is to make bakery products less 'more-ish'. A number of respondents mentioned that there is another route to overcome these challenges. This route is to make a gradual reduction of bakery-product fat contents over an extended period, in small increments. The effects of this approach would be to minimise any changes to product quality, and also allow consumers the time to adjust to slightly different products. Long term, the fat content of bakery products could be reduced in this way. The problem, however, is that without some obvious benefit, it is unlikely that the supply chain will adopt this approach.

\section{Economic}

It is clear from the earlier comments that the reduction of fat in bakery products will have economic implications. Most directly, either changing the balance of standard ingredients, or introducing new ones will both affect the cost of the RFBP. Generally, the comments indicated that the recipe cost is likely to rise, since most other ingredients are more expensive than fat and that some specialised ingredients are expensive in absolute terms. Also there is no single ingredient available to replace fat, so that a 'cocktail' may have to be used. Inevitably this situation will also affect the rate of development of a new RFBP. The exception here is butter, which is expensive relative to other ingredients. Replacement of butter may actually reduce recipe costs, but as discussed previously, there are technical difficulties in doing so and, at this time, it does not appear to be feasible.

Other costs are associated with the increased development time needed for RFBP. For example, there could be possible capital expenditure to modify production lines, small production runs with a loss of economy of scale, extra staff training in modified production techniques, more expensive packaging costs due to smaller quantities being ordered and more wastage in production.

Actual extra cost has not been quantified and will also depend on the RFBP being made, and the level of technical and production challenge.

\section{What level of knowledge exists to help develop reduced-fat bakery products?}

A key area of questioning concerned the knowledge and ingredients available to assist in the development of RFBP. In this area there was a high level of agreement between 
respondents, whichever part of the supply chain they represented.

The general view was that the overall knowledge of ingredient functionality and requirements needed to reduce fat contents was quite low to very low. Know-how exists, but is either fragmented or difficult to locate, or it exists within the technical functions of large companies and, for commercial reasons, is not shared. A number of respondents believe that the level of knowledge is higher in the USA, since the market for RFBP is more highly developed.

Where there is know-how it is thought to be in the area of rebalancing and reformulating recipes to lower fat levels rather than choosing other ingredients for their functionality. A creative approach has been the inclusion of fruit pieces or pastes to create new textures and a variety of flavours, i.e. in terms of constituents, and the replacement of fat with sugars, starches and gums.

Most parts of the supply chain look to the ingredient suppliers for knowledge, suitable ingredients and problem solving. This process does not seem to be working well for various reasons. In general, there is a high expectation that suppliers will understand most about their ingredients. Very often a supplier will develop an ingredient for a particular application and understand how it works through detailed technical studies. The problem arises, however, when new applications are tested. What usually happens is that a producer or retailer will ask for an ingredient to reduce fat levels in a bakery product and is given something which worked in another type of system. If, as often happens, it fails, neither supplier or customer understand why and no solution can be offered.

The overall feeling, then, is that there are many claims for ingredients that will lower fat levels in bakery products, but there are few successes. One producer said 'We are on our own', and this view seemed to reflect those of a number of respondents. Some suppliers commented that when they referred technical problems back to their own research and development centres the response was disappointing. It was considered that the priority for research and problem solving for RFBP was low and they did not always respond positively.

The view was unanimous that there is no single ingredient that can replace fat, nor are there any ingredients that offer universal applications for RFBP. This situation means that product developers have to use mixtures of various functional ingredients without being clear about their interactions and synergies.

The retailer view was that with too many ingredients available, a lot of development time is spent in testing whether they worked. Inevitably, there were a lot of failures and disappointments. When failures do occur it is most likely that, because of the pressures on time, developers will simply move on to another project rather than spend even more time in trying to find another solution.

The view emerged that short-term deadlines for product development are not realistic for RFBP because of the technical challenges, and that a more strategic approach to their development is needed. In a commercial environment this approach is very difficult, and many respondents expressed the view that this development work has to be done in corporate research centres, research associations and universities. Suppliers also noted that they are unlikely to commit large resources to develop new ingredients unless a sufficiently clear market opportunity could be identified.

Most respondents admitted to having failures in the development of RFBP. It is not surprising that many respondents expressed the wish to have a single fat substitute which could be widely used in the development of new RFBP and reduce the amount of development work needed.

\section{How do you see consumer reaction to reduced-fat bakery products?}

This set of questions was designed to probe the developers' view of consumer needs, expectations and behaviour. Although this approach is not a substitute for direct questioning of consumers, its aim was to understand how the product developers gauge consumer attitudes.

\section{Quality}

Respondents believe that consumers have certain expectations of product quality. RFBP, in order to be totally acceptable, must match standard-fat alternatives in all aspects of quality. The further the deviation from this standard, the less it is likely that purchase will occur.

The strong view was that consumers rarely buy nutritional benefits alone; the main driving force for purchase is the liking for the quality of a product, and these quality attributes are often related to the properties which fat gives, for example, mouthfeel, succulence and flavour. Expectations are even higher for luxury or indulgence products (cream cakes, gateaux etc.) and the view was that consumers will not compromise quality at all on these products, they would simply buy something else instead.

Looking forward, respondents believe that if the quality of RFBP could be improved to match standard products, there would be a much greater acceptance and frequency of purchase. It may be that there is a problem with the consumer perception of RFBP because a number of early products were of insufficiently high quality. A typical attitude would then be 'I've tried them, they're no good'. On the positive side it was noted that in general consumers, and maybe the younger age profile, are getting used to eating lighter lower-fat products in other areas, e.g. dairy, meat and snackfoods, and that this factor may help with the acceptance of RFBP.

\section{Claims}

This relates to the claims made for RFBP on the packaging, and links to other consumer expectations, particularly on price, quality and benefits. Some respondents see reducedor low-fat claims as a deterrent to purchase, in that consumers believe that the quality will be lower than that for the standard product, and they have to decide whether to compromise quality for health. The danger is that they may try a product once, on this basis, but often will not make a repeat purchase, which leads to declining sales.

As reported earlier, although there may be a fat claim on the pack, it is believed that sometimes this claim is confused 
with energy reduction and weight control. There may be two distinct types of RFBP buyer, the 'calorie' counter and the fat reducer.

Product claims probably trigger the (unconscious) question: 'what's in this product for me'; of course, there must be an answer on the pack or no purchase will be made. Similarly, consumption of the product must also provide an answer, or there will be no repeat purchase.

\section{Price and benefits}

There was a strong view from producers and retailers that the selling price of RFBP should be very similar to that of a standard product. Consumers will not pay 'more for less', unless the health benefit is very strong. There was a minority view that it is possible to charge premium prices for RFBP, but it was felt that this approach would create a niche market. Prices charged for RFBP can be higher, but the key here is to create a range which is not based on a standard product. Of course, the question is then: how do we know that the price is higher if there is no comparison. The true answer will be in the difference between costs and price, i.e. the profit margin. This point was seen as being important, since this pricing strategy is more likely to generate income that can be invested in longer-term development of the RFBP market.

\section{Behaviour}

More detailed coverage of this aspect has been made separately in a consumer study, but the respondents' view of consumer behaviour is valid within the context of this project. It is believed that the overall picture is complex, and that it is the behaviour of the population as a whole that is being considered here. In other words, there are trends in opposite directions. Whilst there does seem to be an enduring consumer interest in fat intake and health, which is reflected by the growth in the RFBP market, at the same time there is a counter trend for snacking and grazing which is based on the consumption of products of higher fat contents. Respondents were not clear whether the snackers and grazers were also the fat reducers. If so, an increase in snacking and grazing could lead to growth in consumption of RFBP to redress the balance and reduce the feeling of guilt.

The conflict between indulgence and guilt continues. Luxury products represent the former, whereas purchase of RFBPs is seen as a good thing, making us feel less guilty and more virtuous. A number of respondents mentioned that they believe that consumers may eat more reduced-fat biscuits than standard-fat biscuits, because they contain less fat, and whilst feeling more virtuous, may have actually increased their fat intake from this source.

A further specific point was raised about some types of crackers, since these products are often eaten with toppings. The effects of reducing fat content have already been described, and lower-fat crackers tend to be drier and harder. This factor may lead the consumer to increase the amount of topping, e.g. spreading more butter or margarine, or adding more cheese. Ironically, again this action may lead to an increase in fat consumption. The thought-provoking question was raised: would increasing the fat content in crackers, which will create a better texture, lead to lower fat intakes?

\section{What is the long-term future for reduced-fat baking products?}

There were differing views about the current and future potential for the RFBP market. Looking backwards, there are some RFBP which have been on sale for over 5 years, but there has been little organic growth. The key to this lack of growth has been the development and launch of new RFBP. However, the majority view was that the current market is increasing, interest from consumers and retailers is high and there are many new products in the development pipeline. This situation will lead to short-term market growth.

The longer-term situation is less predictable. Again, the majority view is that there is a long-term (10-year) market for RFBP, but its size is difficult to estimate since it will depend on a number of factors. These include:

the extent to which industry understands consumer needs and behaviour;

RFBP quality, improvements will increase market potential;

availability of new ingredients and technologies for fat reduction;

the ability to command premium prices;

a change in nutritional priorities.

There are factors that may limit market growth. Apart from a lack of quality improvement, the biggest threat is the extent to which RFBP will displace the sales of standard products. One retailer mentioned that it was unlikely that they would stock two of everything, i.e. the choice would be to sell the RFBP or the standard product. Another factor, even if both products were to be sold, is the extent to which the reduced-fat version would gradually replace the standard product and become the new norm. In this situation it may be that products with even lower fat contents would then be sought.

Some respondents gave the view that the market was close to peaking, as it has in the USA, and that without further stimulus, will always be a niche. However, it was agreed that the most positive trend is the appearance of bakery products that do not have standard-fat alternatives but appear to contain lower amounts of fat. A number of ranges are now on the market, and they have a turnover in excess of $£ 100$ million per annum. These ranges may genuinely increase the market because there are no standard products to displace, pricing is not linked to a standard product, and variety within the range is constantly increasing.

\section{References}

Department of Health (1991) The Health of the Nation. Department of Health Command Paper no. 1523. London: H.M. Stationery Office.

Food Advisory Committee (1989) Guidelines for the Use of Certain Nutritional Claims in Labelling and Advertising. London: H.M. Stationery Office. 
Food Advisory Committee (1999) Guidelines for the Use of Certain Nutritional Claims in Labelling and Advertising. London: The Stationery Office.
Ministry of Agriculture, Fisheries and Food (1998) National Food Survey. London: The Stationery Office. 